ARTICLE

DOI: $10.1038 / \mathrm{s} 41467-018-06964-\mathrm{x}$

\title{
A homozygous loss-of-function mutation leading to CYBC1 deficiency causes chronic granulomatous disease
}

Gudny A. Arnadottir (1) ', Gudmundur L. Norddahl', Steinunn Gudmundsdottir', Arna B. Agustsdottir?',

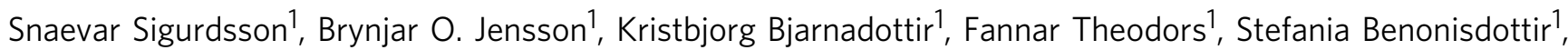
Erna V. Ivarsdottir (1) 1,2, Asmundur Oddsson (1) 1, Ragnar P. Kristjansson', Gerald Sulem?', Kristjan F. Alexandersson', Thorhildur Juliusdottir ${ }^{1}$, Kjartan R. Gudmundsson? ${ }^{1}$, Jona Saemundsdottir?, Adalbjorg Jonasdottir ${ }^{1}$, Aslaug Jonasdottir ${ }^{1}$, Asgeir Sigurdsson ${ }^{1}$, Paolo Manzanillo', Sigurjon A. Gudjonsson', Gudmundur A. Thorisson (1) 1, Olafur Th. Magnusson', Gisli Masson', Kjartan B. Orvar ${ }^{3,4}$, Hilma Holm? , Sigurdur Bjornsson 3,4, Reynir Arngrimsson 5,6, Daniel F. Gudbjartsson (1) 1,2, Unnur Thorsteinsdottir 1,6, Ingileif Jonsdottir ${ }^{1,6}$, Asgeir Haraldsson ${ }^{6,7}$, Patrick Sulem (i) ${ }^{1} \&$ Kari Stefansson ${ }^{1,6}$

Mutations in genes encoding subunits of the phagocyte NADPH oxidase complex are recognized to cause chronic granulomatous disease (CGD), a severe primary immunodeficiency. Here we describe how deficiency of $\mathrm{CYBC1}$, a previously uncharacterized protein in humans (C17orf62), leads to reduced expression of NADPH oxidase's main subunit (gp91phox) and results in CGD. Analyzing two brothers diagnosed with CGD we identify a homozygous loss-of-function mutation, p.Tyr2Ter, in CYBC1. Imputation of p.Tyr2Ter into 155K chipgenotyped Icelanders reveals six additional homozygotes, all with signs of CGD, manifesting as colitis, rare infections, or a severely impaired PMA-induced neutrophil oxidative burst. Homozygosity for p.Tyr2Ter consequently associates with inflammatory bowel disease (IBD) in Iceland $\left(P=8.3 \times 10^{-8} ; \mathrm{OR}=67.6\right)$, as well as reduced height $\left(P=3.3 \times 10^{-4} ;-8.5 \mathrm{~cm}\right)$. Overall, we find that $C Y B C 1$ deficiency results in $C G D$ characterized by colitis and a distinct profile of infections indicative of macrophage dysfunction.

\footnotetext{
${ }^{1}$ deCODE Genetics/Amgen, Inc., Reykjavik, Iceland. ${ }^{2}$ School of Engineering and Natural Sciences, University of Iceland, Reykjavik, Iceland. ${ }^{3}$ Department of Internal Medicine, Landspitali University Hospital, Reykjavik, Iceland. ${ }^{4}$ The Medical Center, Glaesibae, Reykjavik, Iceland. ${ }^{5}$ Department of Genetics and Molecular Medicine, Landspitali University Hospital, Reykjavik, Iceland. ${ }^{6}$ Faculty of Medicine, University of Iceland, Reykjavik, Iceland. ${ }^{7}$ Children's Hospital Iceland, Landspitali University Hospital, Reykjavik, Iceland. These authors contributed equally: Gudny A. Arnadottir, Gudmundur L. Norddahl. Correspondence and requests for materials should be addressed to P.S. (email: patrick.sulem@decode.is) or to K.S. (email: kari.stefansson@decode.is)
} 
C hronic granulomatous disease (CGD) is a rare primary immunodeficiency characterized by severe recurrent bacterial and fungal infections, along with manifestations of chronic granulomatous inflammation ${ }^{1}$. The incidence of the disease varies significantly worldwide, from around 1 in 200,000 in North America and Europe, to 1 in 70,000 in the Israeli Arab population ${ }^{2}$. CGD results from an impaired ability of phagocytes to mount a burst of reactive oxygen species (ROS) in response to pathogens ${ }^{2}$. The production of ROS is catalyzed by a multiprotein enzymatic complex, known as the NADPH oxidase complex $^{3}$. A majority of CGD patients carry pathogenic genotypes in one of five genes encoding subunits of the phagocyte NADPH oxidase; either X-linked recessive mutations in $C Y B B$, or autosomal recessive mutations in $C Y B A, N C F 1, N C F 2$, or $N C F 4^{2,4-6}$. Patients with $\mathrm{X}$-linked recessive mutations in $C Y B B$ are generally recognized to have the most severe disease course with earlier age at onset, whereas patients with autosomal recessive mutations in NCF1 show a significantly higher age at onset $^{1,2,7}$. In a recent review of NCF4 mutations, the clinical presentation of NCF4-deficient patients is described as being even more distinct, resembling a mild, atypical form of $\mathrm{CGD}^{6}$.

For the purpose of searching for associations between variants in the sequence and phenotypes, we have whole-genome sequenced (WGS) $37 \mathrm{~K}$ Icelanders and genotyped $155 \mathrm{~K}$, a large fraction of the Icelandic population (11 and $46 \%$ of $338 \mathrm{~K}$, respectively $)^{8-10}$. This dataset allows for accurate detection of genotypes down to a frequency of $0.01 \%$ in all $155 \mathrm{~K}^{8,9}$. Moreover, by serving as a population reference, the set is proving instrumental for genetic analysis of rare diseases in the clinical setting $8,11,12$.

Through WGS of two brothers diagnosed with CGD, we identify a homozygous loss-of-function mutation, p.Tyr2Ter, in CYBC1 (previously C17orf62). In our existing set of sequenced and chip-genotyped Icelanders we find six additional homozygous individuals. Genotype-based recall confirms that all eight homozygotes have signs of CGD, manifesting as colitis, rare infections, or a severely impaired PMA-induced neutrophil oxidative burst. We show that homozygosity for p.Tyr2Ter results in complete loss of CYBC1, and reduced expression of gp91 phox, NADPH oxidase's main subunit, leading to an impaired oxidative burst. Our results indicate that $\mathrm{CYBC1}$ is essential for successful formation of the NADPH oxidase complex, likely by acting as a chaperone. An excess of colitis in the group of homozygotes, as well as a distinct profile of infections, suggests that CYBC1 deficiency has a more pronounced effect on macrophages. In summary, by leveraging an extensive population database we elucidate the role of a previously uncharacterized gene in humans and identify a novel cause of CGD.

\section{Results}

Analysis of two brothers diagnosed with CGD. We were presented with two Icelandic brothers diagnosed with CGD (individuals $A$ and $B$, Fig. 1a and Table 1), to search for the causal mutation through WGS. The brothers were initially diagnosed with Crohn's disease (CD), at 7 and 9 years of age, after developing severe diarrhea with intestinal biopsies showing granulomatous lesions compatible with CD. The younger brother had developed episodes of acute lymphadenitis and inflammation of the orbit at the ages of 5 and 6 years, respectively. Both brothers experienced recurrent bacterial infections, including infections around the nose and an active wound infection in the mouth, from which the opportunistic bacterium Burkholderia cepacia was cultured. B.cepacia is known to cause infections in immunocompromised hosts, in particular CGD patients ${ }^{4}$. At this stage, the combined clinical, histological, and bacteriological evidence led to a suspicion of CGD. A formal CGD diagnosis was subsequently confirmed for both brothers based on PMA-induced neutrophil oxidative burst tests at the time (Fig. 1b). The number of infections reported for the brothers was nonetheless somewhat less than what would be expected in X-linked CGD patients. The brothers' gastrointestinal symptoms did not respond to conventional treatment for Crohn's disease, and were the main reason for them undergoing hematopoietic stem cell transplantation (HSCT) at 17 (individual A) and 18 (individual B) years of age. The older brother (individual B) died of post-HSCT complications; whereas, the younger brother (individual A) was successfully transplanted in 2010 and has been symptom-free since then (see Supplementary Note 1 for full clinical description).

We sequenced the whole genomes of the two brothers (DNA samples taken pre-HSCT), hereafter referred to as the probands, their three unaffected siblings and parents (see pedigree Fig. 1a, and Methods section). We found no rare coding or splice-site mutations in the five genes known to harbor mutations causing CGD (all five genes were well-covered in the probands' sequence data, Supplementary Table 1). Previously, we had identified a known mutation in $C Y B A^{13}$ in Iceland (NP_000092.2:p. Arg90Trp, MAF $=0.58 \%)$, under a recessive mode of inheritance. We found five Icelanders homozygous for that mutation (out of $155 \mathrm{~K}$ chip-genotyped), two were diagnosed with CGD, and three had a clinical profile consistent with CGD (Supplementary Table 2). Importantly, WGS followed by Sanger sequencing verified the absence of this CYBA mutation from the two probands. We subsequently expanded our analysis to the coding and splicing regions of all RefSeq genes $(n=18,570)$. Due to the rarity of the disease and high penetrance of known CGD mutations we focused on rare genotypes, defined using our reference set of the Icelandic population (37,260 individuals WGS to a median depth of $38 \times$ ). We selected heterozygous genotypes with a minor allele frequency (MAF) below $0.05 \%$, excluding genotypes carried by either of the two unaffected parents, and homozygous/compound heterozygous genotypes where variants had a MAF below 2\%, including both X-linked and autosomal genotypes. We found two genotypes fulfilling these criteria, in genes not previously known to cause Mendelian disease. One of these, a homozygous missense mutation in GCGR encoding the human glucagon receptor (NM_000160.3:c.449G >A; NP_000151.1: p.Ser150Asn; hg38 position chr17:81,811,277; MAF $=0.33 \%)$, was dismissed based on lack of biological relevance (Supplementary Note 2). The other corresponds to a predicted complete knockout, a homozygous stop-gained mutation, p.Tyr2Ter, in CYBC1 (NM_001033046.3:c.6C>G; NP_001028218.1:p.Tyr2Ter; hg38 position chr17:82,449,249; MAF $=0.76 \%$ ). Prior to our application for the gene symbol $C Y B C 1$, it was known under the placeholder symbol C17orf62. The brothers are the only members of their family homozygous for CYBC1 p.Tyr2Ter, their parents and two unaffected siblings are heterozygous carriers, and their third sibling a non-carrier (all confirmed by Sanger sequencing). CYBC1 shares $89 \%$ amino acid sequence identity with the murine bc017643 which was recently shown to be essential for ROS production ${ }^{14}$, consistent with the reduced ROS production described for CGD patients. Both neutrophils and BM-derived macrophages from $b c 017643$-knockout mice showed a highly impaired oxidative burst in response to a range of stimuli, including PMA. Accordingly, bc017643-knockout mice (-/-) showed high susceptibility to bacterial infections, evidenced by Listeria monocytogenes and Salmonella enterica serovar Typhimurium infections ${ }^{14}$. Interestingly, while macrophages from the $b c 017643$-knockout mice had a similar ROS deficit as macrophages from gp91phox/Cybb-deficient mice, neutrophils from the $b c 017643$-knockout mice showed some ROS generation whereas neutrophils from the gp91 phox/Cybb-deficient mice did $\operatorname{not}^{14}$. 

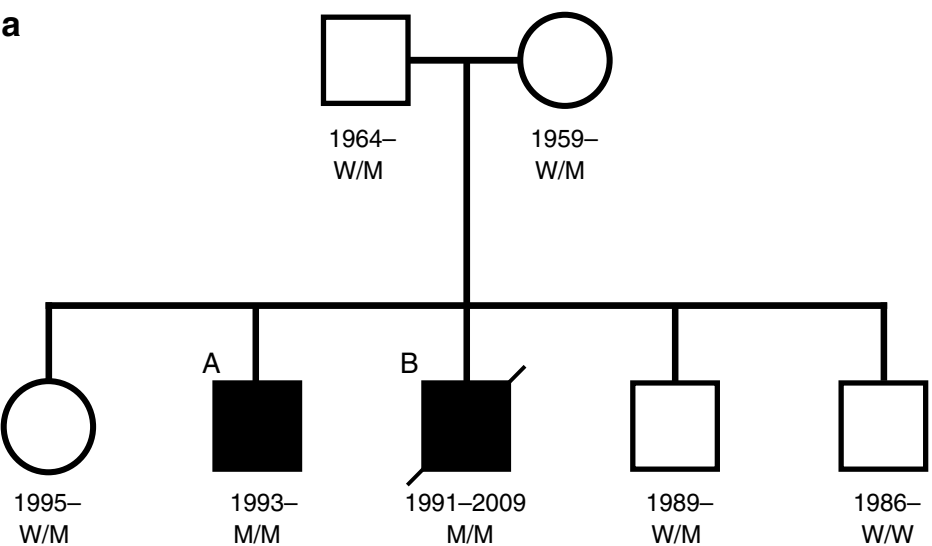

b

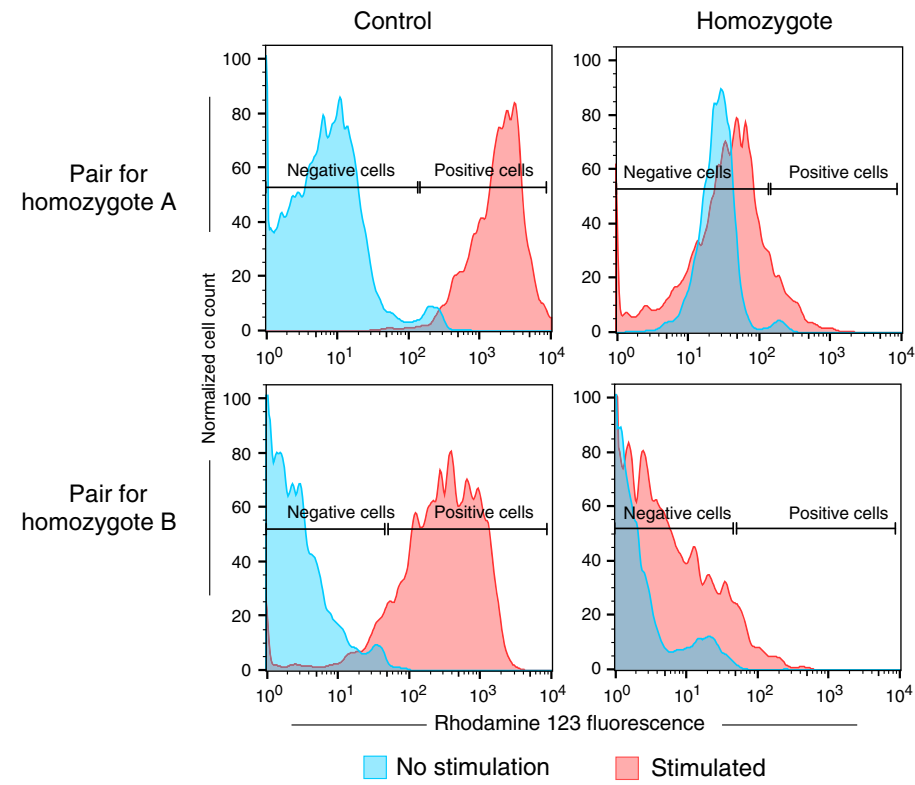

C

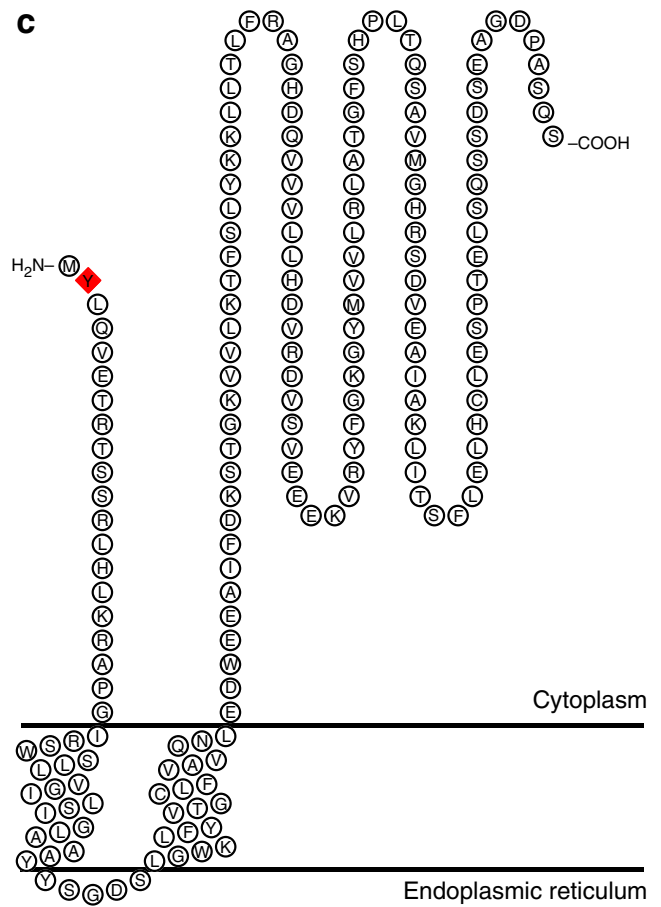

Fig. 1 Pedigree and burst test results for the two probands, and the CYBC1 protein. a Pedigree of the two CGD brothers showing their CYBC1 p.Tyr2Ter genotypes. Squares represent males, circles represent females, and a slashed symbol indicates a deceased individual. Filled symbols represent affected individuals; the two affected are referred to as individuals $A$ and B in the manuscript. The genotype of the p.Tyr2Ter mutation (NP_001028218.1:p.Tyr2Ter; NM_001033046.3:c.6C>G; hg38 position chr17:82,449,249) is indicated with $M$ and W, M representing the mutated allele and W the wild type. M/M therefore indicates homozygous status, W/M indicates heterozygous status and W/W a non-carrier. $\mathbf{b}$ Neutrophil oxidative burst test for the two CGD brothers homozygous for CYBC1 p.Tyr2Ter (individuals A and B) and their controls, test was performed pre-HSCT. Left panel shows fluorescent peaks for unstimulated and PMA stimulated neutrophils from the controls, and the right panel shows peaks for unstimulated and PMA stimulated neutrophils from the two homozygous brothers. Negative and positive cells are defined by setting a gate for unstimulated cells. Neutrophils from individuals $A$ and $B$ failed to generate an oxidative burst equivalent to their controls, their respective stimulation indices were $\mathrm{SI}_{\mathrm{A}}=1.34$ and $\mathrm{SI}_{\mathrm{B}}=2.50$. $\mathbf{c}$ Topological prediction of CYBC1 (NP_001028218.1) ${ }^{28}$. CYBC1 is predicted to be a transmembrane protein, spanning the lipid bilayer via two transmembrane regions (aa 21-39 and aa 45-63). A red diamond represents the p.Tyr2Ter mutation at the second amino acid of the protein

The availability of population level data allowed us to search for other individuals homozygous for CYBC1 p.Tyr2Ter. Out of a learning set of 28,075 WGS Icelanders we detected two other homozygous individuals, as well as 372 heterozygous carriers of the mutation. This allowed for accurate imputation (Methods section) of the mutation into our set of $155 \mathrm{~K}$ chip-genotyped Icelanders based on long-range haplotype sharing ${ }^{8}$ (imputation information $=0.99$ ). Through imputation we found four additional individuals homozygous for p.Tyr2Ter, and confirmed their genotypes with Sanger sequencing.
Colitis and rare infections in p.Tyr2Ter homozygotes. To investigate the consequences of CYBC1 p.Tyr2Ter, we searched for pathological manifestations in these six additional homozygotes and found that three had colitis, like the two probands, and had received a diagnosis of inflammatory bowel disease (IBD) (Table 1). Intestinal inflammation is a common manifestation of CGD and around $40 \%$ of CGD patients develop CD-like problems ${ }^{15}$. Of the three additional homozygotes suffering from colitis, two had a confirmed granulomatous colitis (individuals $\mathrm{C}$ and F). Individual $C$ was initially diagnosed with ulcerative colitis 


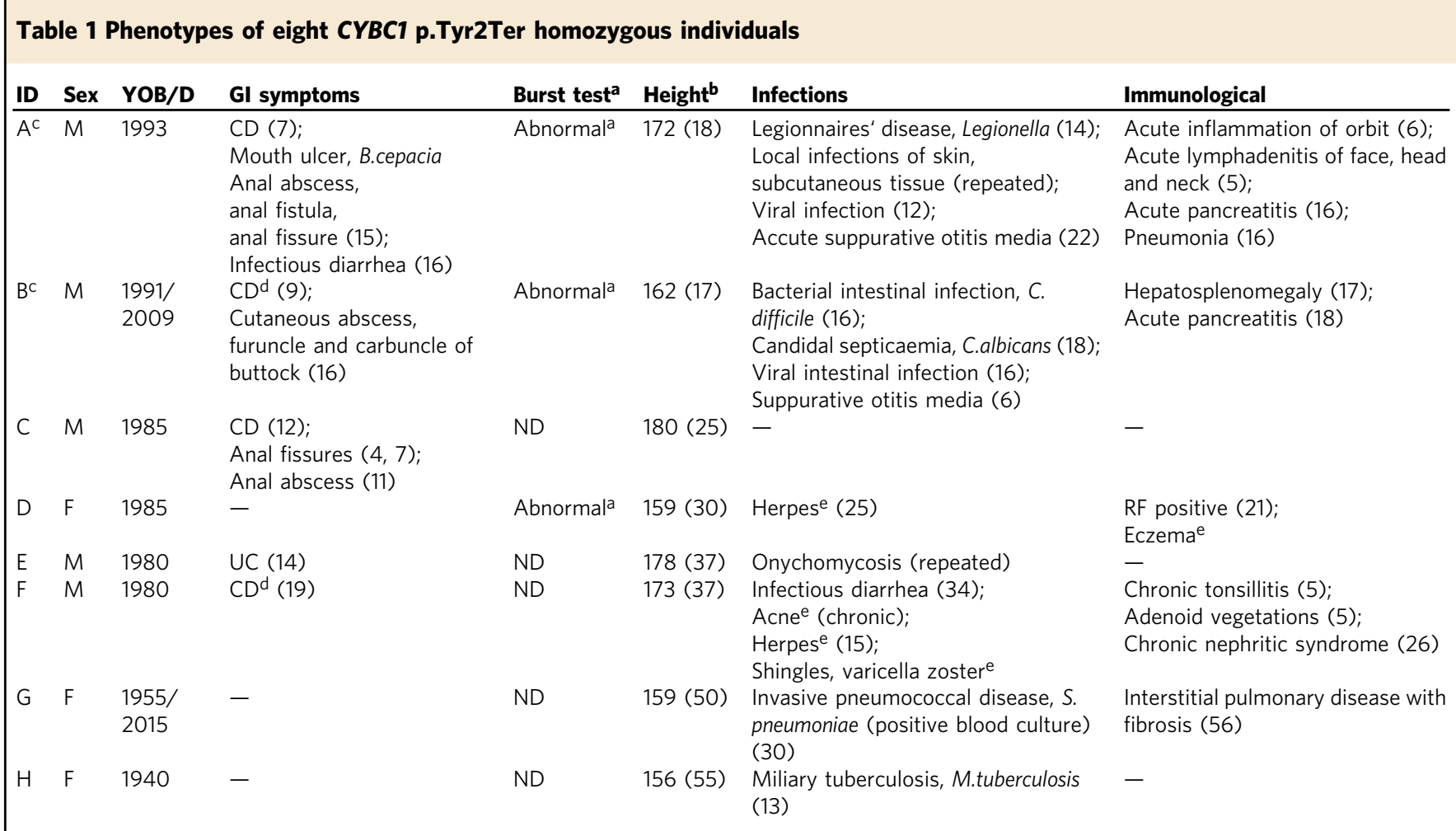

Figures in parentheses () denote age at diagnosis or measurement, in years

YOB/D year of birth/death (rounded by 5 years for individuals $C-H$ ), Gl gastrointestinal, $C D$ Crohn's disease, UC ulcerative colitis, CGD chronic granulomatous disease, ND not determined, $R F$ rheumatoid YOB/D year of birth/death (rounded by 5 years for individuals $\mathrm{C}-\mathrm{H}$ ), Gl gastrointestinal, CD Crohn's disease, UC ulc
factor

aBurst test for individual A and B was performed in 2008, burst test for individual D was performed in 2017

All heights are given in $\mathrm{cm}$. Average and SD for height of Icelandic males and females is $178.8 \pm 6.9 \mathrm{~cm}$ and $165.6 \pm 6.3 \mathrm{~cm}$, respectively 35

Individuals $\mathrm{A}$ and $\mathrm{B}$ are brothers, presented in Fig. 1

Individuals $B$ and $F$ underwent total colectomy

eSelf-reported phenotypes (via an online questionnaire)

(UC), receiving a $\mathrm{CD}$ diagnosis only after undergoing total colectomy that revealed extensive granulomatous inflammation of the colon. The third individual, individual E, was diagnosed at 14 years of age with total colitis. Repeated biopsies from colon did not yield granulomatous changes. However, even though granulomas were not observed in individual $\mathrm{E}$, his clinical course could be considered to better fit CD than UC. Notably, granulomatous changes are unconfirmed in the majority of CGD patients with colitis $^{16}$. Furthermore, diffuse colitis involving all regions of the colon, as seen for individual E, could be considered characteristic of the gastrointestinal involvement in $\mathrm{CGD}^{16}$. As expected, $\mathrm{p}$. Tyr2Ter associates with IBD in Iceland under the recessive model $\left(P=8.3 \times 10^{-8}\right.$; OR $(95 \% \mathrm{CI})=67.6(14.5,315.5) ; n_{\text {cases }}=2429$; $n_{\text {controls }}=338,647$; likelihood ratio test; Supplementary Table 3). Limiting to chip-typed individuals, we found that heterozygous carriers of p.Tyr2Ter were not at a significantly higher risk of IBD than non-carriers $(P=0.31$; OR $(95 \% \mathrm{CI})=1.21(0.84,1.75)$; $n_{\text {cases }}=1701 ; n_{\text {controls }}=135,106$; likelihood ratio test).

To gather additional clinical and biological information we attempted genotype-based recall of p.Tyr2Ter homozygotes for phenotyping. We obtained additional hospital-based information (including discharge diagnoses and laboratory measurements, see Table 1 and Methods section), and found that seven out of eight homozygotes had experienced severe and/or early-onset bacterial or fungal infections (including the two probands). Individual D was the only one not to have reported any severe infections, at 30 years of age. In patients with CGD, microbial catalase is traditionally recognized as the most important virulence factor in infections ${ }^{17}$. Consistent with this, many of the infections noted in the homozygous individuals were caused by catalase-positive pathogens, including a mucous membrane infection by Burkholderia cepacia ${ }^{2}$, candidal septicemia (Candida albicans ${ }^{18}$ ), Legionnaires' disease (Legionella ${ }^{19}$ ), and miliary tuberculosis (Mycobacterium tuberculosi ${ }^{20}$ ). The rarity of most of these infections is notable, only 61 occurrences of Legionella infections have been reported in Iceland between 1997 and 2017, and only 19 individuals have been diagnosed with candidal septicemia over the same period ${ }^{21}$. Thus, it is noteworthy that individual A developed both Legionnaires' disease and candidal septicemia. Similarly, only 55 occurrences of miliary tuberculosis have been noted in Iceland, including individual $\mathrm{H}$ (out of 11,438 tuberculosis cases, diagnosed between 1901 and 198922). Individual $G$ had invasive pneumococcal disease at 30 years of age caused by Streptococcus pneumoniae ${ }^{7}$, and later developed interstitial pulmonary fibrosis, a noted complication of the exaggerated inflammatory response in $\mathrm{CGD}^{2,23,24}$.

In terms of microbe spectrum, the overall infectious profile of the homozygous individuals could be considered somewhat unusual for CGD. The invasive fungal sepsis by C.albicans, and the miliary tuberculosis by M.tuberculosis are characteristic of CGD, as well as the pneumonia episodes reported for individual A (at 14 years of age, registered as Legionnaires' disease, and again at 16 years of age). However, the pathogens cultured from the pneumonia episodes are only sporadically reported in CGD (Legionella ${ }^{1,25}$ and S.pneumoniae ${ }^{7}$ ), and the absence of some of the most frequently encountered pathogens in CGD, such as Staphylococcus aureus and Aspergillus species, is noteworthy ${ }^{2,7}$. 


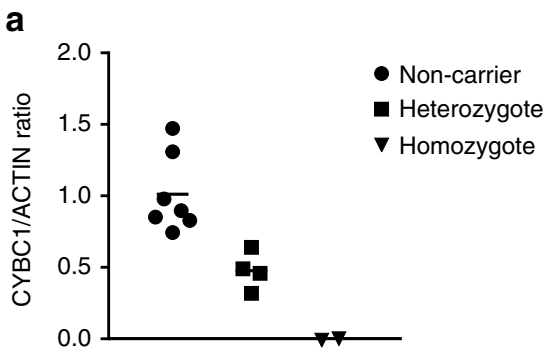

b
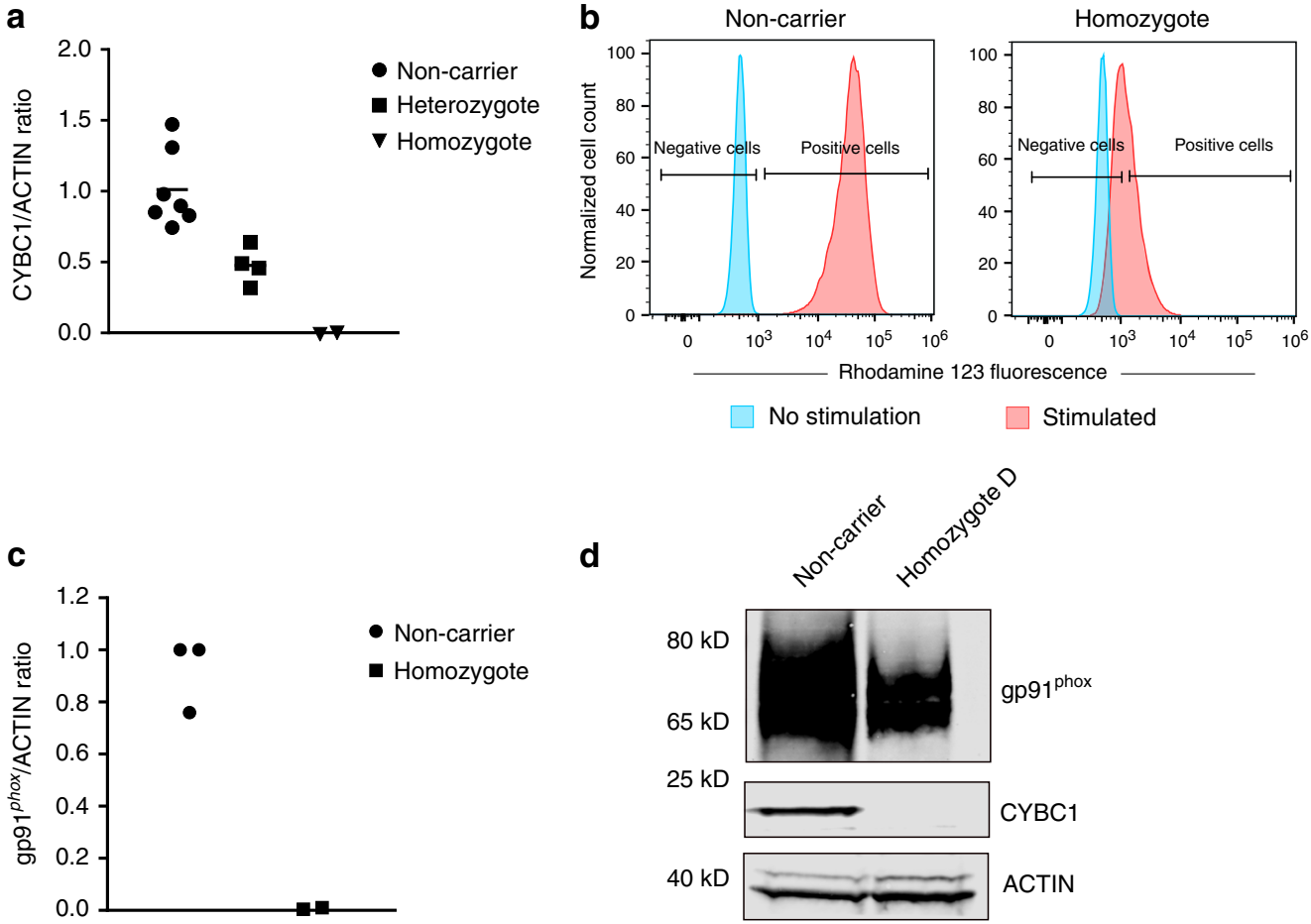

d

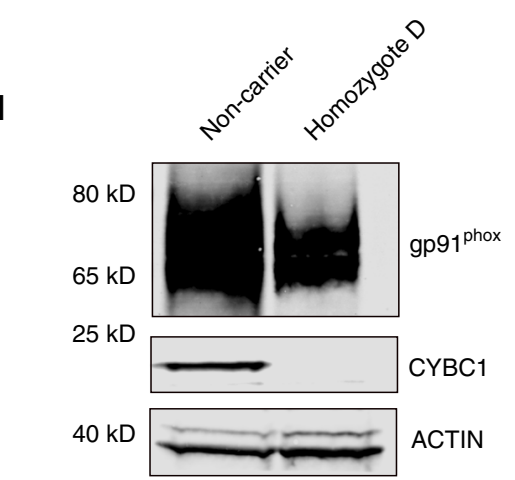

Fig. 2 Effect of p.Tyr2Ter on CYBC1, the neutrophil oxidative burst, and gp91phox. a CYBC1 protein expression, relative to ACTIN, in lymphocytes from CYBC1 p.Tyr2Ter homozygous individuals D and F (triangles), four heterozygous carriers (squares), and matched (age and sex) non-carriers (circles). CYBC1 expression was not detected in the homozygous individuals in contrast to their matched non-carriers, and was reduced by $53 \%$ in the heterozygous carriers, compared to matched non-carriers. The analysis was performed by western blot (shown in Supplementary Fig. 3 and 4), where ACTIN was used as a loading control. b Neutrophil oxidative burst test for CYBC1 p.Tyr2Ter homozygous individual D and her matched (age and sex) non-carrier. Left panel shows fluorescent peaks for unstimulated and PMA stimulated neutrophils from the non-carrier, and the right panel shows peaks for unstimulated and PMA stimulated neutrophils from homozygous individual D. Negative and positive cells are defined by setting a gate for unstimulated cells. Neutrophils from individual $D$ failed to generate an oxidative burst equivalent to her matched non-carrier, the stimulation index for homozygous individual $D$ was $S I_{D}=$ 2.35. c Protein expression of gp91phox, relative to ACTIN, in monocyte-derived macrophages from CYBC1 p.Tyr2Ter homozygotes D and F (squares) and their matched (age and sex) non-carriers (circles). gp91phox expression was absent in the two homozygotes in contrast to their matched non-carriers. The analysis was performed by western blot (shown in Supplementary Fig. 5), where ACTIN was used as a loading control. d CYBC1 and gp91phox expression in fresh neutrophils from CYBC1 p.Tyr2Ter homozygous individual D and her matched (age and sex) non-carrier. CYBC1 expression was not detected in the homozygous individual in contrast to the non-carrier. gp91phox expression was $\sim 50 \%$ lower in the homozygote than her matched non-carrier. The analysis was performed by western blot, and ACTIN was used as a loading control

In addition to the two probands, we performed WGS for the six other CYBC1 p.Tyr2Ter homozygotes. The five individuals with gastrointestinal involvement were analyzed for variants previously known to cause monogenic $\mathrm{IBD}^{15,26}$, and the absence of such variants confirmed. Additionally, no known or expected pathogenic variants were detected in other known CGD genes in any of the eight p.Tyr2Ter homozygotes. Consistent with the effect of other known CGD genotypes on stature ${ }^{4}$ we also found that homozygosity for p.Tyr2Ter associates with reduced height $\left(P=3.3 \times 10^{-4}\right.$; effect $(95 \% \mathrm{CI})=-1.24 \mathrm{SD} \quad(-1.92,-0.56) /$ $-8.5 \mathrm{~cm}$; likelihood ratio test; Supplementary Tables 3 and 4 ). Taking all of the above into consideration, we conclude that the clinical presentation of seven out of eight homozygous individuals (all but $\mathrm{D}$ ) is suggestive of CGD.

Functional consequences of p.Tyr2Ter. CYBC1 encodes a 187 amino acid protein (NP_001028218.1, canonical as defined by RefSeq), highly expressed in whole blood ${ }^{14,27}$. It is predicted to be a transmembrane protein, spanning the lipid bilayer via two transmembrane regions (Fig. 1c)28. The p.Tyr2Ter mutation introduces a premature termination codon at the second amino acid of the protein, preventing translation of the full-length protein. We observed no reduction in CYBC1 mRNA expression in white blood cells from heterozygous carriers of p.Tyr2Ter $\left(n_{\text {carriers }}=29 ; n_{\text {non-carriers }}=2430 ; P=0.86\right.$; Effect $(95 \% \mathrm{CI})=0.03$ SD $(-0.36,0.30)$, Supplementary Fig. 1$)$, nor did we observe any signs of allelic imbalance when assessing allele-specific expression (Supplementary Fig. 2). This indicates that p.Tyr2Ter does not lead to nonsense-mediated mRNA decay (NMD), consistent with NMD being less efficient when premature termination codons occur in such close proximity to the start codon ${ }^{29}$. Rather, we expect p.Tyr2Ter to result in a lack of product at the protein level. We investigated the possibility of an alternative start codon in $C Y B C 1$ producing a functional protein. The closest codon for methionine following the same reading frame as p.Tyr2Ter occurs in exon 6 (out of 7 exons). The non-coding mRNA corresponding to this start codon (NR_036518, expressed in whole blood ${ }^{27}$ ) encodes a sequence of 63 amino acids, lacking the two membranespanning domains of CYBC1.

To study the direct effect of p.Tyr2Ter at the protein level, we performed western blot analysis on available lymphocytes from four heterozygous carriers of p.Tyr2Ter, revealing 53\% less CYBC1 protein than in matched non-carriers (Fig. 2a). Additionally, through genotype-based recall we obtained blood 
samples from two p.Tyr2Ter homozygotes (individuals D and F, Table 1). We found no trace of the full-length CYBC1 protein in lymphocytes from the two homozygotes (Fig. 2a).

Chaperone for cytochrome b-245. The oxidative burst consists of a rapid production and release of ROS, a process catalyzed by the NADPH oxidase complex ${ }^{3}$. The phagocyte NADPH oxidase is composed of six subunits, three cytosolic components ( $\mathrm{p} 47^{\mathrm{phox}}$, p67phox , and $\mathrm{p} 40^{\text {phox }}$ ), a regulatory G-protein ( $\left.\mathrm{Rac} 1 / \mathrm{Rac} 2\right)$ and an essential membrane-bound heterodimer (gp91phox-p22phox), known as cytochrome b-245, which mediates the transfer of electrons to oxygen ${ }^{3}$. The dimerization of gp91phox and $\mathrm{p} 22^{\text {phox }}$ is a relatively inefficient process, and as unassembled monomers the two proteins quickly undergo degradation ${ }^{14,30,31}$. Neutrophils and bone marrow (BM)-derived macrophages from bc017643-knockout mice had markedly reduced expression of both gp91phox and p22 phox , despite expressing normal mRNA levels for these proteins, most likely due to the role of bc017643 as a chaperone in the dimerization of cytochrome b-245. The cytosolic components of NADPH oxidase (p47phox , p67 ${ }^{\text {phox }}$, and $\mathrm{p} 40^{\text {phox }}$ ) were not differentially expressed in $b c 017643$-deficient mice ${ }^{14}$. To determine whether a similar role could be expected for CYBC1 in humans, we measured gp91phox in monocyte-derived macrophages and neutrophils from p.Tyr2Ter homozygotes. We detected no gp91phox in monocyte-derived macrophages from homozygous individuals $\mathrm{D}$ and $\mathrm{F}$ (Fig. 2c), in contrast to matched non-carriers, and a $\sim 50 \%$ reduction in gp91 phox levels in neutrophils from individual D (neutrophils were not available from individual $\mathrm{F}$, Fig. 2d). Furthermore, we were able to test and confirm an impaired PMA-induced oxidative burst in neutrophils from homozygous individual D (Fig. 2b), thereby providing independent confirmation of an impaired oxidative burst in a p.Tyr2Ter homozygote (as observed previously for the two probands, Fig. 1b). Individual D is therefore positive for the test widely used as a diagnostic test for CGD without having, to our knowledge, developed colitis or chronic infections at age 30 (Table 1 and Methods section). Our findings, together with the prior observation in mice, suggest a chaperone role for CYBC1 in the dimerization of gp91 phox and $\mathrm{p} 22^{\text {phox }}$ in humans. We therefore renamed the gene $C Y B C 1$, for cytochrome b-245 chaperone 1 .

The p.Tyr2Ter mutation is absent from 138,632 exomes/ genomes in a large multi-ethnic public database (gnomAD), and no other coding homozygous loss-of-function mutation is listed in the canonical transcript on gnomAD ${ }^{32}$ (Supplementary Table 5). Nonetheless, we came across a homozygous missense mutation in CYBC1, p.Asp43Asn (NM_001033046.3:c.127G >A, hg38 position chr17:82,447,580), in a single case in a recent publication from Saudi Arabia ${ }^{33}$. Although the mutation was classified as a missense, we identified its location to be at the exonic splice junction where it is predicted to disrupt correct splicing of the mRNA ${ }^{34}$. Interestingly, the individual homozygous for that mutation had symptoms compatible with CGD, including lymphadenopathy, hepatosplenomegaly, recurrent fever, and growth retardation ${ }^{33}$.

\section{Discussion}

In summary, among $155 \mathrm{~K}$ chip-genotyped Icelanders we identified eight homozygous for a rare loss-of-function mutation, $\mathrm{p}$. Tyr2Ter, in CYBC1 (previously known as the uncharacterized C17orf62). The eight homozygotes show signs compatible with CGD, either gastrointestinal symptoms, rare infections, and/or a biochemically proven oxidative burst defect.

Impaired expression of gp91phox in p.Tyr2Ter homozygous individuals, the oxidative burst defect, and prior evidence in knockout mice all point to $\mathrm{CYBC1}$ as an essential factor for successful formation of the NADPH oxidase complex. CYBC1 is known to co-localize and interact with gp91phox in the endoplasmic reticulum (ER) ${ }^{14,35}$, where it likely acts as a chaperone for dimerization of gp91phox and p22phox (cytochrome b-245). Although no definitive conclusion can be drawn from only one individual, the difference in expression of gp91phox between monocyte-derived macrophages (absent) and neutrophils (50\% reduced) from homozygous individual D is noteworthy, and suggests the presence of more than one chaperone for cytochrome b-245 in the neutrophil lineage. Consequently, mechanisms more specific to macrophages, such as the exaggerated proinflammatory responses seen in CGD patients ${ }^{36}$, may be a more prominent phenotype in CYBC1-deficient individuals. The complete absence of $g p 91^{\text {phox }}$ from macrophages is also in line with some of the infections observed in the p.Tyr2Ter homozygous individuals, like M.tuberculosis ${ }^{37}$, Legionella ${ }^{38}$, and C. albicans $^{39}$, as well as the increased susceptibility of $b c 017643$ knockout mice to S.Typhimurium and L.monocytogenes infections ${ }^{14}$. CYBC1 deficiency thus appears to predispose to a distinct spectrum of microbes that can be considered more specific to macrophages. Supportive of this, a prior study of two distinct $C Y B B$ mutations causing infections restricted to mycobacteria demonstrated an impaired oxidative burst in macrophages but not in neutrophils ${ }^{40}$.

We expect $C Y B C 1$ p.Tyr2Ter to have reached its frequency in Iceland (carried by about 1 in 70) through the founder effect ${ }^{8}$. The founder effect allows such mutations to reach a considerable frequency in the bottlenecked/founder population of Iceland, resulting in homozygotes in high-enough numbers to confer statistical power for discovery of disease associations ${ }^{8,41}$. We have identified eight p.Tyr2Ter homozygous Icelanders in our dataset, comprising $155 \mathrm{~K}$ chip-genotyped individuals, and would expect 19 homozygotes in the entire Icelandic population $(338 \mathrm{~K}$ individuals). Overall, we observe thirteen Icelanders with a genotype causing signs compatible with CGD, eight homozygous for CYBC1 p.Tyr2Ter and five for CYBA p.Arg90Trp. This amounts to a predicted disease prevalence of 1 in 12,000 in Iceland, much higher than reported for CGD in the literature ${ }^{2}$. In addition to the founder effect in Iceland, this higher overall prevalance of CGD may be explained by the fact that in some instances the causative genotypes lead to a mild phenotype resulting in underdiagnosis of the disease. In Iceland however, we are simply assessing prevalence by identifying pathogenic genotypes.

Our results indicate that CYBC1-deficient individuals have some residual ROS production, similar to what has been reported for CGD patients with NCF1 biallelic mutations ${ }^{42}$. Residual ROS production has been shown to correlate with a milder clinical presentation, and improved overall survival in CGD depending on early detection and treatment of the disease ${ }^{42}$. Our findings therefore underline the value of using genotypes for the identification of previously undiagnosed cases. Out of the 13 Icelanders with a CGD genotype, only the two probands and two of the CYBA p.Arg90Trp homozygotes were, to our knowledge, diagnosed with CGD prior to the discovery of CYBC1. Reporting of the genotypes of the remaining nine individuals to their treating physicians is already proving important for their clinical followup and treatment.

The set of $155 \mathrm{~K}$ chip-genotyped Icelanders used for our study consists of individuals healthy enough to have survived up to the time of recruitment, with the vast majority over 18 years old at the time of sample acquisition (mean age at recruitment is 46 years). The set is therefore biased toward adults, and the identification of genotypes from such a set can be expected to reveal greater phenotypic variability than recruitment based on clinical presentation alone, as is illustrated by our study. The mean age at onset of symptoms for the five p.Tyr2Ter homozygous 
individuals who had gastrointestinal manifestations is 12.2 years, and deviates somewhat from X-linked recessive CGD patients, the vast majority of whom are diagnosed before 5 years of age ${ }^{2}$. The CYBC1 p.Tyr2Ter homozygotes also contrast patients with NCF4 biallelic mutations in terms of loss-of-neutrophil NADPH oxidase activity. The CYBC1-deficient individuals have strongly impaired PMA-induced neutrophil oxidative burst (stimulation indices lower than 3, as in X-linked CGD ${ }^{43,44}$, see Fig. $1 \mathrm{~b}$ and Fig. 2b), whereas NCF4-deficient patients have normal or mildly impaired PMA-induced neutrophil oxidation ${ }^{5,6}$. We recognize that for full understanding of the molecular mechanism of CYBC1 deficiency, a more detailed analysis of NADPH oxidase activity is required, such as assessment of particle-induced ROS production, as well as experiments with other cell types.

Previously, we and others have identified and described a large set of rare complete human knockouts ${ }^{45-48}$. By systematically investigating homozygous loss-of-function carriers, we are beginning to understand how some of these mutations relate to diseases and other traits. Through genotype-based recall of individuals homozygous for CYBC1 p.Tyr2Ter, we were able to confirm the loss-of-function effect of p.Tyr2Ter by verifying the absence of the CYBC1 protein. Moreover, this allowed us to demonstrate that complete loss of CYBC1 leads to a reduced neutrophil oxidative burst in the absence of pathogenic mutations in known components of the NADPH oxidase complex. Hitherto, pathogenic mutations in five genes encoding subunits of the phagocytic NADPH oxidase complex were known to cause CGD. We have shown that $C Y B C 1$ represents a novel player in the NADPH pathway and the sixth CGD gene.

\section{Methods}

Study population and generation of genetic dataset. Our approach to wholegenome sequencing, genotyping, long-range phasing, and imputation has been described in detail in previous publications ${ }^{8,9}$. In brief, 28,075 Icelanders participating in various disease projects at deCODE genetics were whole-genome sequenced using standard TrueSeq methodology (Illumina) to a median depth of $37 \times$. Further, 155,250 Icelanders have been genotyped with Illumina microarrays (chip-genotyped), and genotype probabilites for untyped relatives have been calculated based on Icelandic genealogy. Genotypes of sequence variants identified through sequencing (SNPs and indels) have been imputed into all chip-typed Icelanders and their close relatives (familial imputation).

An extension of this learning set of 28,075 WGS Icelanders are 9185 Icelanders more recently recruited and sequenced, amounting to a set of 37,260 WGS Icelanders used for the current study. These 37,260 Icelanders were all sequenced by the same method as previously described, to a median depth of $38 \times{ }^{8,9}$.

All participating individuals who donated blood or buccal tissue samples, or their guardians, provided written informed consent. All sample identifiers were encrypted in accordance with the regulations of the Icelandic Data Protection Authority. Personal identities of the participants and biological samples were encrypted by a third-party system approved and monitored by the Icelandic Data Protection Authority. Years of births/deaths in Table 1 of individuals not recruited through clinical sequencing (individuals $\mathrm{C}-\mathrm{H}$ ) are rounded to five years (pedigrees are provided in Supplementary Fig. 6). The study was approved by the Data Protection Authority (ref. 2013030423/PS/--, with amendments) and the National Bioethics Committee (ref. VSN 12-121, VSNb2012070013/03.12), that also reviewed and approved the protocol, methodology and all documents presented to the participants. All methods were performed in accordance with the relevant guidelines and regulations.

Imputation. Imputation is a method for inferring genotypic status of variants that have not been directly genotyped. Imputation of untyped variants into the mix of typed variants is now routine in human genetics ${ }^{8}$. The extensive genealogical information available for the Icelandic population, along with deCODE's longrange phasing of a large set of genotyped Icelanders ${ }^{8,49}$ has increased imputation accuracy and speed by removing uncertainty in phasing. In general, a haplotype can be imputed into an untyped individual if two genotyped relatives share a long haplotype that is identical by descent (IBD), and the genealogy indicates that the path of IBD sharing goes through the individual ${ }^{49}$.

The informativeness of genotype imputation is estimated by the ratio of the variance of imputed expected allele counts and the variance of the actual allele counts:

$$
\frac{\operatorname{Var}(\mathrm{E}(\theta \mid \text { chip data }))}{\operatorname{Var}(\theta)},
$$

where $\theta$ is the haplotype allele count. $\operatorname{Var}(\mathrm{E}(\theta \mid$ chip data $))$ was estimated by the observed variance of the allele imputations and $\operatorname{Var}(\theta)$ was estimated by $p(1-p)$, where $p$ is the allele frequency. This metric has the property of being 0 when the imputation is completely uninformative (the same value is always imputed) and 1 when the imputation is fully informative. Asymptotically, the expectation of the metric equals the $r^{2}$ between the imputed alleles and the true genotypes.

Whole-genome sequencing of the two probands and their family. Genomic DNA (gDNA) was isolated from blood samples taken pre-HSCT from the two affected brothers and from buccal samples taken in 2016 from their parents and three siblings. A request for whole-genome sequencing (WGS) was sent through an in-house laboratory information management system (LIMS). gDNA was registered and isolated by an in-house core facility (Biological Materials facility). Samples were delivered to the Genome Sequencing Laboratory in a barcoded 96well tray format and stored at $4{ }^{\circ} \mathrm{C}$ until use. Samples were prepared for sequencing using the TruSeq PCR-free library preparation kits from Illumina, and sequenced on Illumina instruments to an average genome-wide coverage of $39 \times$. Details of the sample preparation, paired-end sequencing, read processing and alignment and filtering of resulting BAM files have been described in previous publications ${ }^{8,9}$.

Variant calling, annotation, and filtering. Variants were called using version 2.3-9 of the Genome Analysis Toolkit (GATK $)^{50}$, reads were called with GATK's HaplotypeCaller, version 2014.4-3.3.0-0-ga3711aa, using joint calling. In our analysis of the WGS data of the two probands we focused on SNPs and small indels (shorter than 20 base pairs) at coding and splicing regions in their genomes, as annotated by release 80 of the Variant Effect Predictor ${ }^{51}$, using RefSeq gene annotations. We assessed the frequency of observed sequence variants using (1) our set of 37,260 whole-genome sequenced Icelanders ${ }^{8,9}$ at a median depth of $38 \times$ and gnom $\mathrm{AD}^{32}$, and (2) 155,250 chip-genotyped Icelanders with 32.5 million imputed variants. We defined rare autosomal recessive genotypes as homozygous or compound heterozygous sequence variants, each with a minor allele frequency lower than $2 \%$ in our set and gnom $\mathrm{AD}^{32}$. We defined rare autosomal dominant genotypes as variants with a minor allele frequency lower than $0.05 \%$ in our set and gnomAD.

Association testing. We tested for association between sequence variants and phenotypes under a recessive mode of inheritance, as previously described ${ }^{52,53}$. We used logistic regression to test for association with binary phenotypes, adjusting for sex, age, and county within Iceland. Quantitative phenotypes were tested using a linear mixed model implemented in BOLT-LMM ${ }^{54}$. Height measurements were corrected for year of birth, sex, and age at measurement, using a rank-based inverse normal standardization. A total of 31.6 million variants were tested for association under a multiplicative model. For the recessive analysis, there were 19.2 million variants with homozygotes for the minor allele in the dataset. All of the variants that were tested had imputation information over 0.8 . The threshold for genomewide significance was corrected for multiple testing with a weighted Bonferroni adjustment using as weights the enrichment of variant classes with predicted functional impact among association signals ${ }^{55}$. The significance threshold then becomes $2.5 \times 10^{-7}$ for high-impact variants (including stop-gained, frameshift, splice acceptor or donor), $5.0 \times 10^{-8}$ for moderate-impact variants (including missense, splice-region variants and in-frame indels), $4.5 \times 10^{-9}$ for low-impact variants, $2.3 \times 10^{-9}$ for DNase I hypersensitivity site (DHS) variants and $7.5 \times 10$ -10 for remaining variants.

Phenotypic information. We performed phenotypic assessment of the eight individuals homozygous for CYBC1 p.Tyr2Ter by (1) examining a large set of existing clinical, biological, and genealogical information and (2) obtaining novel phenotypic information through an extensive health study of the Icelandic population. The study consists of a 4 -h visit with a medical examination, interview and collection of biological samples. For individuals participating in the study we are able to review, through a two-way encryption system, hospital-based information, including hospital discharge diagnoses and laboratory tests. This study allows us to perform deeper phenotyping for individuals targeted by their genotype (genotypebased recall). We have attempted genotype-based recall for a set of $\sim 13,000$ loss-offunction homozygous individuals, based on WGS and imputation, and controls ${ }^{45}$

Sanger sequencing. The $C Y B C 1$ p.Tyr2Ter mutation was submitted to Sanger sequencing for confirmation of a homozygous genotype in the eight individuals detected by WGS and chip-genotyping. The position of the p.Tyr2Ter mutation was also sequenced by the Sanger method in the five family members of the two probands (Fig. 1a). The CYBA p.Arg90Trp mutation was also submitted to Sanger sequencing for all eight CYBC1 p.Tyr2Ter homozygous individuals. Primers for Sanger sequencing were designed using the Primer 3 software ${ }^{56}$. Following PCR, 
cycle sequencing reactions were performed in both directions on MJ Research PTC-225 thermal cyclers, using the BigDye Terminator Cycle Sequencing Kit v3.1 (Life Technologies) and Ampure XP and CleanSeq kits (Agencourt) for cleanup of the PCR products and cycle sequencing reactions. Sequencing products were loaded onto the 3730 XL DNA Analyzer (Applied Biosystems) and analyzed with the Sequencher 5.0 software (GeneCodes Corporation).

Neutrophil oxidative burst in homozygous individual D. We performed a neutrophil oxidative burst test for $C Y B C 1$ p.Tyr2Ter homozygous individual D (Table 1). The neutrophil oxidative burst was measured on a fresh whole-blood sample from individual D with a Phagoburst kit (BD biosciences, \#341058) used according to the manufacturer's instructions. In short, a venous blood sample was collected in a lithium heparin tube (Vacuette, \#455084) and thereafter $100 \mu \mathrm{L}$ of blood were aliqoted and placed on ice for $10 \mathrm{~min}$. Samples were then stimulated with PMA for exactly $10 \mathrm{~min}$ at $37^{\circ} \mathrm{C}$, followed by $10 \mathrm{~min}$ incubation with the fluorogenic substrate dihydrorhodamine (DHR) 123 at $37^{\circ} \mathrm{C}$, cell lysis and fixation. Samples were analyzed on a flow cytometer (FACSCalibur, BD and Attune Nxt, Thermo Fisher). Gating strategy is shown in Supplementary Fig. 7.

Blood cell isolation and macrophage differentiation. For neutrophil isolation, fresh whole blood was mixed with Lympholyte-poly (Cedarlane \#CL5070) at a 1:1 ratio, following the manufacturer's instructions. Thereafter, the sample was centrifuged at $500 \times g$ for $35 \mathrm{~min}$ and the polymorphonuclear cell layer was extracted by pipetting and resuspended in PBS $+2 \%$ FBS. For PBMC isolation, $25 \mathrm{~mL}$ of fresh whole blood was added to $15 \mathrm{~mL}$ Ficoll-Paque PLUS (GE Healthcare, \#17-1440-03) in a SepMate tube (STEMCELL Technologies, \#15450). Then, the sample was centrifuged at $1200 \times \mathrm{g}$ for $10 \mathrm{~min}$. The plasma layer was discarded and the PBMC layer transferred to a fresh tube followed by washes in PBS $+2 \%$ FBS.

Untouched monocytes were purified from PBMC preparations using the Monocyte Isolation Kit II (Miltenyi, \#130-091-153) following the manufacturer's instructions. Labeled samples were run on MS columns, and both the flow-through (consisting of untouched monocytes), and column bound lymphocytes (mainly B-, T-, and NK-cells) were collected as well for western blot analysis.

Monocyte-derived macrophages were generated by culturing monocytes for 6 days in X-vivo medium (Lonza, \#04744Q) supplemented with Glutamax (Thermo Fisher, \#35050038), Sodium Pyruvate (Thermo Fisher, \#11360039), 50 ng/mL M-CSF (Miltenyi, \#130-091-153). Fresh medium was added to cells on day 3 and on day 5 media were aspirated and replaced with the same media with $50 \mathrm{ng} /$ mL IFN-g (Thermo Fisher, \#PHC4031). Cells were collected on day 6 for western blot analysis.

Western blot analysis. Cell pellets were lysed in $100 \mu \mathrm{L}$ RIPA buffer (150 mM $\mathrm{NaCl}, 1 \%$ Triton X-100, $0.5 \%$ sodiumdeoxycholate, $0.1 \%$ SDS, $50 \mathrm{mM}$ Tris, $\mathrm{pH} 8$ ) with halt protease and phosphatase inhibitor (Thermo Fisher, \#78445) and incubated for $10 \mathrm{~min}$ on ice. Following brief sonication, samples were spun at $14,000 \times \mathrm{g}$ for $15 \mathrm{~min}$ at $4^{\circ} \mathrm{C}$. The supernatant was transferred to a fresh tube and the protein content of lysates was determined by BCA assay (Thermo Fisher, \#23227). Samples were mixed with NuPAGE LDS sample buffer (Thermo Fisher, \#B0007) and reducing agent (Thermo Fisher, \#B0009), heated for $10 \mathrm{~min}$ at $70{ }^{\circ} \mathrm{C}$ and loaded on a $4-12 \%$ BT bolt plus gel (Thermo Fisher, \#NW04120BOX). The gel was equilibrated for $5 \mathrm{~min}$ in $20 \% \mathrm{EtOH}$ and transferred onto a nitrocellulose membrane (Thermo Fisher, \# IB23002) with iBlot 2 (Thermo Fisher) running the P0 program. Following the transfer, membranes were re-hydrated in PBS for $5 \mathrm{~min}$ and after that blocked in Odyssey blocking buffer (LI-COR, \#92740000). Blots were incubated with primary antibodies overnight at $4{ }^{\circ} \mathrm{C}$ in Odyssey blocking buffer + $0.05 \%$ Tween 20. Antibodies used were, anti-gp91phox at 1:1000 (Santa Cruz, \#sc130543), anti-C17orf62 at 1:1000 (Atlas Antibodies, \#HPA045696), anti-beta-actin at 1:2500 (Biotechne, \#MAB8929), anti-beta-actin at 1:5000 (Cell Signaling, \#4970), anti-mouse IgG (H+L) IRDye $800 \mathrm{CW}$ at 1:10,000 (LI-COR, \#926-32212), antirabbit IgG $(\mathrm{H}+\mathrm{L})$ IRDye680RD at 1:10,000 (LI-COR, \#926-68073), and anti-rabbit IRDye $800 \mathrm{CW}$ at 1:10,000 (LI-COR, \#925-32211). Membranes were scanned with Odyssey CLx and subsequent analysis performed in the image studio software (LICOR). Uncropped blots are provided as Supplementary Fig. 8 and 9.

\section{Data availability}

Our previously described Icelandic population whole-genome sequence data ${ }^{9}$ have been deposited at the European Variant Archive under accession PRJEB15197. The authors declare that the data supporting the findings of this study are available within the article, its supplementary information files and upon request. The HGNC accepted our application for the gene symbol CYBC1 instead of the placeholder C17orf62 on February 26th 2018, listed under accession 28672.

Received: 29 January 2018 Accepted: 8 October 2018

Published online: 25 October 2018

\section{References}

1. Song, E. et al. Chronic granulomatous disease: a review of the infectious and inflammatory complications. Clin. Mol. Allergy 9, 10 (2011).

2. Arnold, D. E. \& Heimall, J. R. A review of chronic granulomatous disease. Adv. Ther. 34, 2543-2557 (2017).

3. Segal, B. H., Leto, T. L., Gallin, J. I., Malech, H. L. \& Holland, S. M. Genetic, biochemical, and clinical features of chronic granulomatous disease. Medicine 79, 170-200 (2000).

4. Holland, S. M. Chronic granulomatous disease. Clin. Rev. Allergy Immunol. 38, 3-10 (2010)

5. Matute, J. D. et al. A new genetic subgroup of chronic granulomatous disease with autosomal recessive mutations in p40 phox and selective defects in neutrophil NADPH oxidase activity. Blood 114, 3309-3315 (2009).

6. van de Geer, A. et al. Inherited p40phox deficiency differs from classic chronic granulomatous disease. J. Clin. Invest. 128, 3957-3975 (2018).

7. van den Berg, J. M. et al. Chronic granulomatous disease: the European experience. PLoS ONE 4, e5234 (2009).

8. Gudbjartsson, D. F. et al. Large-scale whole-genome sequencing of the Icelandic population. Nat. Genet. 47, 435-444 (2015).

9. Jónsson, $\mathrm{H}$. et al. Whole genome characterization of sequence diversity of 15,220 Icelanders. Sci. Data 4, 170115 (2017).

10. Statistics Iceland (2018). Available at: https://statice.is/statistics/population/ inhabitants/overview/.

11. Arnadottir, G. A. et al. Compound heterozygous mutations in UBA5 causing early-onset epileptic encephalopathy in two sisters. BMC Med. Genet. 18, 103 (2017).

12. Jensson, B. O. et al. COPA syndrome in an Icelandic family caused by a recurrent missense mutation in COPA. BMC Med. Genet. 18, 129 (2017).

13. Rae, J. et al. Molecular analysis of 9 new families with chronic granulomatous disease caused by mutations in CYBA, the gene encoding p22(phox). Blood 96, 1106-1112 (2000)

14. Thomas, D. C. et al. Eros is a novel transmembrane protein that controls the phagocyte respiratory burst and is essential for innate immunity. J. Exp. Med. 214, 1111-1128 (2017).

15. Uhlig, H. H. et al. The diagnostic approach to monogenic very early onset inflammatory bowel disease. Gastroenterology 147, 990-1007.e3 (2014).

16. Schäppi, M. G., Smith, V. V., Goldblatt, D., Lindley, K. J. \& Milla, P. J. Colitis in chronic granulomatous disease. Arch. Dis. Child. 84, 147-151 (2001).

17. Agger, W. A. \& Kowalski, T. J. Chronic granulomatous disease, catalase, and Actinomyces. Clin. Infect. Dis. 50, 1325-1326 (2010).

18. Wysong, D. R., Christin, L., Sugar, A. M., Robbins, P. W. \& Diamond, R. D Cloning and sequencing of a Candida albicans catalase gene and effects of disruption of this gene. Infect. Immun. 66, 1953-1961 (1998).

19. Bandyopadhyay, P., Byrne, B., Chan, Y., Swanson, M. S. \& Steinman, H. M. Legionella pneumophila catalase-peroxidases are required for proper trafficking and growth in primary macrophages. Infect. Immun. 71, 4526-4535 (2003)

20. Zhang, Y., Heym, B., Allen, B., Young, D. \& Cole, S. The catalase-peroxidase gene and isoniazid resistance of Mycobacterium tuberculosis. Nature 358, 591 (1992).

21. The Directorate of Health (2017). Available at: https://www.landlaeknir.is/ smit-og-sottvarnir/smitsjukdomar/sjukdomur/item12522/Legionella.

22. Sveinbjornsson, G. et al. HLA class II sequence variants influence tuberculosis risk in populations of European ancestry. Nat. Genet. 48, 318-322 (2016).

23. Jones, L. B. K. R. et al. Special article: chronic granulomatous disease in the United Kingdom and Ireland: a comprehensive national patient-based registry. Clin. Exp. Immunol. 152, 211-218 (2008).

24. Mahdaviani, S. A. et al. Pulmonary manifestations of chronic granulomatous disease. Expert Rev. Clin. Immunol. 9, 153-160 (2013).

25. Dorman, S. E. et al. Nocardia infection in chronic granulomatous disease. Clin Infect. Dis. 35, 390-394 (2002).

26. Bianco, A. M., Girardelli, M. \& Tommasini, A. Genetics of inflammatory bowel disease from multifactorial to monogenic forms. World J. Gastroenterol. 21, 12296-12310 (2015)

27. GTEx Consortium. The genotype-tissue expression (GTEx) project. Nat. Genet. 45, 580-585 (2013).

28. Käll, L., Krogh, A. \& Sonnhammer, E. L. L. A combined transmembrane topology and signal peptide prediction method. J. Mol. Biol. 338, 1027-1036 (2004).

29. Lindeboom, R. G. H., Supek, F. \& Lehner, B. The rules and impact of nonsense-mediated mRNA decay in human cancers. Nat. Genet. 48, $1112-1118(2016)$ 
30. DeLeo, F. R. et al. Processing and maturation of flavocytochrome b558 include incorporation of heme as a prerequisite for heterodimer assembly. J. Biol. Chem. 275, 13986-13993 (2000).

31. Noubade, R. et al. NRROS negatively regulates reactive oxygen species during host defence and autoimmunity. Nature 509, 235-239 (2014).

32. Lek, M. et al. Analysis of protein-coding genetic variation in 60,706 humans. Nature 536, 285-291 (2016).

33. Monies, D. et al. The landscape of genetic diseases in Saudi Arabia based on the first 1000 diagnostic panels and exomes. Hum. Genet. 136, 921-939 (2017).

34. Desmet, F.-O. et al. Human Splicing Finder: an online bioinformatics tool to predict splicing signals. Nucleic Acids Res. 37, e67 (2009).

35. Laurindo, F. R. M., Araujo, T. L. S. \& Abrahão, T. B. Nox NADPH oxidases and the endoplasmic reticulum. Antioxid. Redox Signal. 20, 2755-2775 (2014).

36. Meissner, F. et al. Inflammasome activation in NADPH oxidase defective mononuclear phagocytes from patients with chronic granulomatous disease. Blood 116, 1570-1573 (2010).

37. Cambier, C. J., Falkow, S. \& Ramakrishnan, L. Host evasion and exploitation schemes of Mycobacterium tuberculosis. Cell 159, 1497-1509 (2014).

38. Newton, H. J., Ang, D. K. Y., van Driel, I. R. \& Hartland, E. L. Molecular pathogenesis of infections caused by Legionella pneumophila. Clin. Microbiol. Rev. 23, 274-298 (2010).

39. Netea, M. G., Joosten, L. A. B., van der Meer, J. W. M., Kullberg, B.-J. \& van de Veerdonk, F. L. Immune defence against Candida fungal infections. Nat. Rev. Immunol. 15, 630-642 (2015).

40. Bustamante, J. et al. Germline CYBB mutations that selectively affect macrophages in kindreds with $\mathrm{X}$-linked predisposition to tuberculous mycobacterial disease. Nat. Immunol. 12, 213-221 (2011).

41. Gudbjartsson, D. F. et al. A frameshift deletion in the sarcomere gene MYL4 causes early-onset familial atrial fibrillation. Eur. Heart J. 38, 27-34 (2017).

42. Kuhns, D. B. et al. Residual NADPH oxidase and survival in chronic granulomatous disease. N. Engl. J. Med. 363, 2600-2610 (2010).

43. Köker, M. Y. et al. Clinical, functional, and genetic characterization of chronic granulomatous disease in 89 Turkish patients. J. Allergy Clin. Immunol. 132, 1156-1163.e5 (2013)

44. Vowells, S. J. et al. Genotype-dependent variability in flow cytometric evaluation of reduced nicotinamide adenine dinucleotide phosphate oxidase function in patients with chronic granulomatous disease. J. Pediatr. 128, 104-107 (1996).

45. Sulem, P. et al. Identification of a large set of rare complete human knockouts. Nat. Genet. 47, 448-452 (2015).

46. Lim, E. T. et al. Rare complete knockouts in humans: population distribution and significant role in autism spectrum disorders. Neuron 77, 235-242 (2013).

47. Saleheen, D. et al. Human knockouts and phenotypic analysis in a cohort with a high rate of consanguinity. Nature 544, 235 (2017)

48. MacArthur, D. G. et al. A systematic survey of loss-of-function variants in human protein-coding genes. Science 335, 823-828 (2012).

49. Kong, A. et al. Detection of sharing by descent, long-range phasing and haplotype imputation. Nat. Genet. 40, 1068-1075 (2008).

50. McKenna, A. et al. The Genome Analysis Toolkit: a MapReduce framework for analyzing next-generation DNA sequencing data. Genome Res. 20, 1297-1303 (2010).

51. McLaren, W. et al. The ensembl variant effect predictor. Genome Biol. 17, 122 (2016).

52. Benonisdottir, S. et al. Epigenetic and genetic components of height regulation. Nat. Commun. 7, 13490 (2016).
53. Styrkarsdottir, U. et al. Whole-genome sequencing identifies rare genotypes in COMP and CHADL associated with high risk of hip osteoarthritis. Nat. Genet. 49, 801-805 (2017).

54. Loh, P.-R. et al. Efficient Bayesian mixed-model analysis increases association power in large cohorts. Nat. Genet. 47, 284 (2015).

55. Sveinbjornsson, G. et al. Weighting sequence variants based on their annotation increases power of whole-genome association studies. Nat. Genet. 48, 314-317 (2016).

56. Koressaar, T. \& Remm, M. Enhancements and modifications of primer design program Primer3. Bioinformatics 23, 1289-1291 (2007).

\section{Acknowledgements}

We wish to thank the family of the two probands, as well as all the other individuals who participated in the study and whose contribution made this work possible.

\section{Author contributions}

G.A.A., G.L.N., S.S., U.T., I.J., P.S., and K.S. designed the study and interpreted the results. G.L.N., S.G., A.B.A., K.B., F.T., and P.M. performed laboratory experiments and interpreted the results. S.A.G., G.M., G.A.T., and D.F.G. developed the methods for data analysis. J.S., K.B.O., H.H., Si.B., R.A., and A.H. carried out or supervised phenotyping. O.Th.M., Ad.J., As.J., and A.S. performed the sequencing. G.A.A., B.O.J., S.B., E.V.I., A O., R.P.K., G.S., K.F.A., T.J., K.R.G., D.F.G., and P.S. performed the statistical and bioinformatics analyses. G.A.A., G.L.N., D.F.G., U.T., I.J., A.H., P.S., and K.S. drafted the manuscript. All authors contributed to the final version of the paper.

\section{Additional information}

Supplementary Information accompanies this paper at https://doi.org/10.1038/s41467018-06964-x.

Competing interests: The authors affiliated with deCODE genetics/Amgen declare a conflict of interest as employees. The remaining authors declare no competing interests.

Reprints and permission information is available online at http://npg.nature.com/ reprintsandpermissions/

Publisher's note: Springer Nature remains neutral with regard to jurisdictional claims in published maps and institutional affiliations.

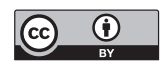

Open Access This article is licensed under a Creative Commons Attribution 4.0 International License, which permits use, sharing, adaptation, distribution and reproduction in any medium or format, as long as you give appropriate credit to the original author(s) and the source, provide a link to the Creative Commons license, and indicate if changes were made. The images or other third party material in this article are included in the article's Creative Commons license, unless indicated otherwise in a credit line to the material. If material is not included in the article's Creative Commons license and your intended use is not permitted by statutory regulation or exceeds the permitted use, you will need to obtain permission directly from the copyright holder. To view a copy of this license, visit http://creativecommons.org/ licenses/by/4.0/.

(c) The Author(s) 2018 